\title{
Permanent draft genome sequence of the gliding predator Saprospira grandis strain Sa g1 (= HR1)
}

\author{
Konstantinos Mavromatis ${ }^{1}$, Olga Chertkov ${ }^{1,2}$, Alla Lapidus ${ }^{1}$, Matt Nolan ${ }^{1}$, Susan Lucas ${ }^{1}$, \\ Hope Tice ${ }^{1}$, Tijana Glavina Del Rio ${ }^{1}$, Jan-Fang Cheng ${ }^{1}$, Cliff Han ${ }^{1,2}$, Roxanne Tapia ${ }^{1,2}$, David \\ Bruce $^{1,2}$, Lynne A. Goodwin ${ }^{1,2}$, Sam Pitluck ${ }^{1}$, Marcel Huntemann ${ }^{1}$, Konstantinos Liolios ${ }^{1}$, \\ Ioanna Pagani ${ }^{1}$, Natalia Ivanova ${ }^{1}$, Natalia Mikhailova ${ }^{1}$, Amrita Pati ${ }^{1}$, Amy Chen ${ }^{3}$, Krishna \\ Palaniappan $^{3}$, Miriam Land ${ }^{1,4}$, Evelyne-Marie Brambilla ${ }^{6}$, Manfred Rohde ${ }^{5}$, Stefan Spring ${ }^{6}$, \\ Markus Göker ${ }^{6}$, John C. Detter ${ }^{1,2}$, James Bristow ${ }^{1}$, Jonathan A. Eisen ${ }^{1,7}$, Victor Markowitz ${ }^{3}$, \\ Philip Hugenholtz ${ }^{1,8}$, Nikos C. Kyrpides ${ }^{1}$, Hans-Peter Klenk ${ }^{6 *}$, and Tanja Woyke ${ }^{1}$ \\ ${ }^{1}$ DOE Joint Genome Institute, Walnut Creek, California, USA \\ ${ }^{2}$ Los Alamos National Laboratory, Bioscience Division, Los Alamos, New Mexico, USA \\ ${ }^{3}$ Biological Data Management and Technology Center, Lawrence Berkeley National \\ Laboratory, Berkeley, California, USA \\ ${ }^{4}$ Oak Ridge National Laboratory, Oak Ridge, Tennessee, USA \\ ${ }^{5}$ HZI - Helmholtz Centre for Infection Research, Braunschweig, Germany \\ ${ }^{6}$ Leibniz Institute DSMZ - German Collection of Microorganisms and Cell Cultures, \\ Braunschweig, Germany \\ ${ }^{7}$ University of California Davis Genome Center, Davis, California, USA \\ ${ }^{8}$ Australian Centre for Ecogenomics, School of Chemistry and Molecular Biosciences, The \\ University of Queensland, Brisbane, Australia
}

*Corresponding authors: Hans-Peter Klenk (hpk@dsmz.de)

Keywords: strictly aerobic, gliding, Gram-negative, mesophilic, chemoorganotrophic, marine, ixotrophy, Saprospiraceae, GEBA

Saprospira grandis Gross 1911 is a member of the Saprospiraceae, a family in the class 'Sphingobacteria' that remains poorly characterized at the genomic level. The species is known for preying on other marine bacteria via 'ixotrophy'. S. grandis strain Sa g1 was isolated from decaying crab carapace in France and was selected for genome sequencing because of its isolated location in the tree of life. Only one type strain genome has been published so far from the Saprospiraceae, while the sequence of strain Sa g1 represents the second genome to be published from a non-type strain of $S$. grandis. Here we describe the features of this organism, together with the complete genome sequence and annotation. The 4,495,250 bp long Improved-High-Quality draft of the genome with its 3,536 protein-coding and 62 RNA genes is a part of the Genomic Encyclopedia of Bacteria and Archaea project.

\section{Introduction}

Strain Sa g1 (= HR1 = DSM $2844=$ ATCC $49590=$ LMG 13157) belongs to the species Saprospira grandis [1,2] in the monospecific genus Saprospira $[2,3]$. The type strain of the species is Lewin $\mathrm{WH}^{\mathrm{T}}(=$ ATCC $23119=$ LMG 10407) [1,3] and is known for its predatory life style when capturing and preying on other bacteria via 'ixotrophy' [2]. Strain Sa g1 was isolated in 1975 from decaying crab carapace in Roscoff, France [4]. The genus name was derived from the Greek adjective sapros, meaning rotten/putrid, and the Latin spira, a coil/spiral, resulting in the Neo-Latin Saprospira, a spiral associated with decaying matter [5]; the species epithet was derived from the Latin adjective grandis, large [5]. Life style and ecological role of members of the species was recently summarized by Saw et al. [6] when they reported the genome sequence of strain Lewin (isolated from La Jolla beach in San Diego; not to be confused with strain Lewin $\mathrm{WH}^{\mathrm{T}}$, the type strain of the species which was also isolated by Lewin, but from a rockpool near high water, Woods Hole). Strain Lewin was the first member of the genus Saprospira to be completely sequenced. Here we present a summary classification and a set of features for $S$. grandis Sa g1, together with the description of the genomic sequencing and annotation. 


\section{Classification and features}

A representative genomic 16S rRNA sequence of strain Sa g1 was compared using NCBI BLAST $[7,8]$ under default settings (e.g., considering only the high-scoring segment pairs (HSPs) from the best 250 hits) with the most recent release of the Greengenes database [9] and the relative frequencies of taxa and keywords (reduced to their stem [10]) were determined, weighted by BLAST scores. The most frequently occurring genera were Saprospira (82.0\%), Aureispira (5.4\%), "Aureospira" (4.8\%), Cytophaga (3.9\%) and Lewinella (3.8\%) (16 hits in total). Regarding the three hits to sequences from members of the species, the average identity within HSPs was 99.4\%, whereas the average coverage by HSPs was $98.6 \%$. Among all other species, the one yielding the highest score was Aureispira maritima (AB278130), which corresponded to an identity of $87.3 \%$ and an HSP coverage of $98.0 \%$. (Note that the Greengenes database uses the INSDC (= EMBL/NCBI/DDBJ) annotation, which is not an authoritative source for nomenclature or classification.) The highestscoring environmental sequence was FJ792500 ('Unexpectedly archaeal species shift between rare and dominant over thousand year time scales carbonate chimney Lost City Hydrothermal Field clone SGYF672'), which showed an identity of 99.2\% and an HSP coverage of $100.3 \%$. The most frequently occurring keywords within the labels of all environmental samples which yielded hits were 'lake' (3.8\%), 'sludg' (2.9\%), 'microbi' (2.8\%), 'mat' $(2.7 \%)$ and 'activ' (2.3\%) (234 hits in total) and correspond to the already known habitats for strains of this species.

Figure 1 shows the phylogenetic neighborhood of $S$. grandis strain Sa g1 in a 16S rRNA based tree. The sequences of the four $16 \mathrm{~S}$ rRNA gene copies in the genome differ from each other by up to one nucleotide, and differ by up to seven nucleotides from the previously published $16 \mathrm{~S}$ rRNA sequence (M58795), which contains 52 ambiguous base calls.

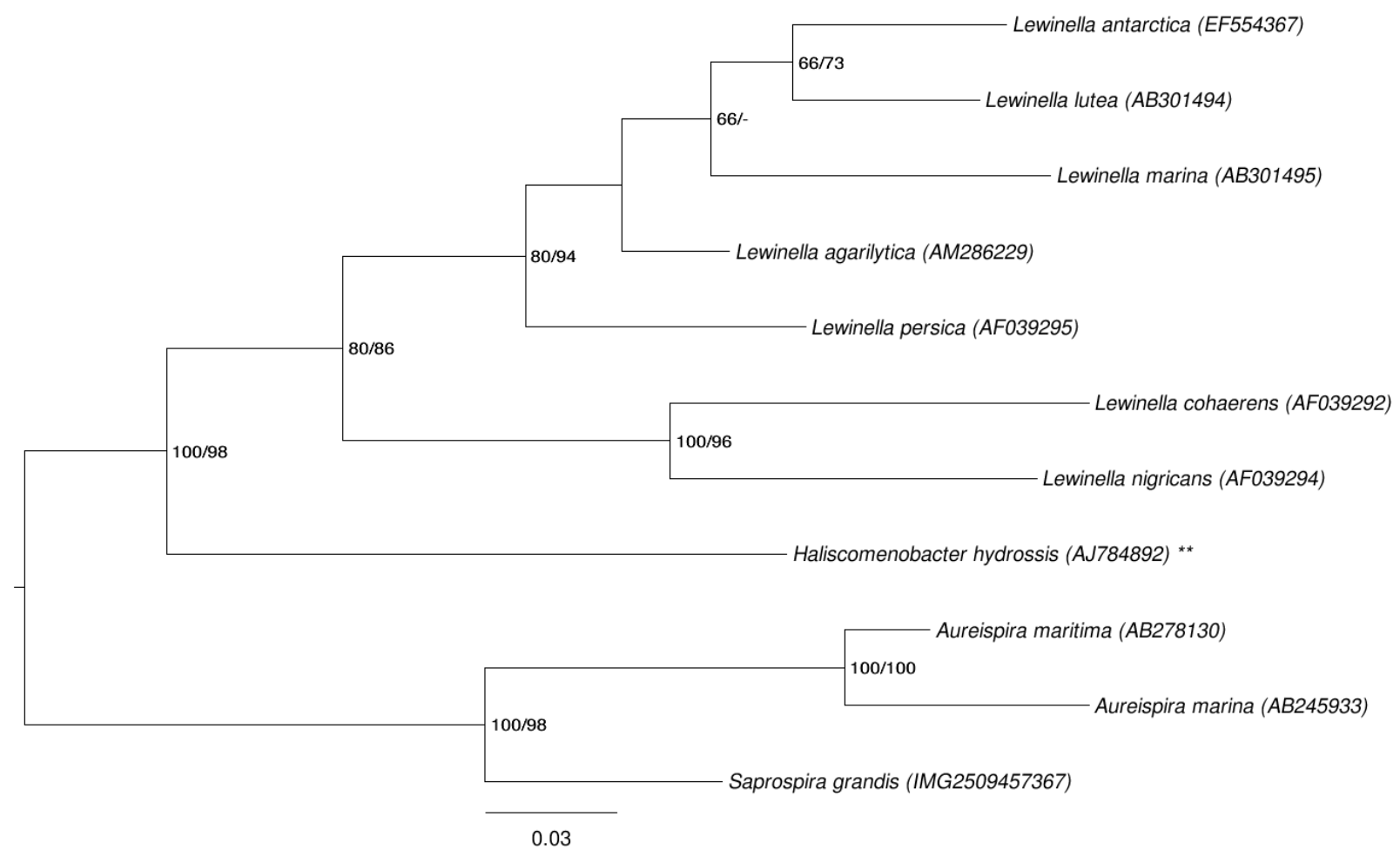

Figure 1. Phylogenetic tree highlighting the position of $S$. grandis relative to the type strains of the other species within the family Saprospiraceae. The tree was inferred from 1,413 aligned characters [11,12] of the 16S rRNA gene sequence under the maximum likelihood (ML) criterion [13]. Rooting was done initially using the midpoint method [14] and then checked for its agreement with the current classification (Table 1). The branches are scaled in terms of the expected number of substitutions per site. Numbers adjacent to the branches are support values from $250 \mathrm{ML}$ bootstrap replicates [15] (left) and from 1,000 maximum parsimony bootstrap replicates [16] (right) if larger than 60\%. Lineages with type strain genome sequencing projects registered in GOLD [17] are labeled with one asterisk, those also listed as 'Complete and Published' with two asterisks [18]. 
General features of $S$. grandis were summarized previously by Saw et al. in the short genome report of strain Lewin [6], and are therefore not repeated here. Individual features of strain Sa g1 are largely unknown due to a lack of relevant publications, as are chemotaxonomical data. A description of the isolation and some morphological features of strain Sa g1 are reported by Reichenbach [4]. Figure 2 shows an electron micrograph of the $S$. grandis Sa g1 cells.

Table 1. Classification and general features of $S$. grandis Sa g1 according to the MIGS recommendations [19] and the Names for Life database [2].

\begin{tabular}{|c|c|c|c|}
\hline MIGS ID & Property & Term & Evidence code \\
\hline & \multirow{8}{*}{ Current classification } & Domain Bacteria & TAS [20] \\
\hline & & Phylum Bacteroidetes & TAS $[21,22]$ \\
\hline & & Class 'Sphingobacteria' & TAS $[21,23]$ \\
\hline & & Order Sphingobacteriales & TAS $[21,24]$ \\
\hline & & Family Saprospiraceae & TAS $[21,25]$ \\
\hline & & Genus Saprospira & TAS $[3,26,27]$ \\
\hline & & Species Saprospira grandis & TAS $[3,26]$ \\
\hline & & Strain Sa g1 & TAS [4] \\
\hline & Gram stain & negative & TAS $[28,29]$ \\
\hline & Cell shape & helical filaments & TAS $[28,29]$ \\
\hline & Motility & via gliding & TAS $[28,29]$ \\
\hline & Sporulation & non-sporulating & NAS \\
\hline & Temperature range & mesophile, $6-47^{\circ} \mathrm{C}$ & TAS $[28,29]$ \\
\hline & Optimum temperature & $25-30^{\circ} \mathrm{C}$ & TAS $[4,28,29]$ \\
\hline & Salinity & seawater & TAS $[28,29]$ \\
\hline \multirow[t]{3}{*}{ MIGS-22 } & Oxygen requirement & strictly aerobe & TAS $[28,29]$ \\
\hline & Carbon source & peptides, proteins & TAS $[28,29]$ \\
\hline & Energy metabolism & chemoorganotroph & TAS $[28,29]$ \\
\hline MIGS-6 & Habitat & marine littoral zone & TAS [28] \\
\hline MIGS-15 & Biotic relationship & free living & TAS [28] \\
\hline \multirow[t]{2}{*}{ MIGS-14 } & Pathogenicity & not reported & \\
\hline & Biosafety level & 1 & TAS [30] \\
\hline MIGS-23.1 & Isolation & decaying crab carapace & TAS [4] \\
\hline MIGS-4 & Geographic location & Roscoff, France & TAS [4] \\
\hline MIGS-5 & Sample collection time & September 1975 & TAS [4] \\
\hline MIGS-4.1 & Latitude & 48.70 & NAS \\
\hline MIGS-4.2 & Longitude & -3.97 & \\
\hline MIGS-4.3 & Depth & not reported & \\
\hline MIGS-4.4 & Altitude & not reported & \\
\hline
\end{tabular}

Evidence codes - IDA: Inferred from Direct Assay (first time in publication); TAS: Traceable Author Statement (i.e., a direct report exists in the literature); NAS: Non-traceable Author Statement (i.e., not directly observed for the living, isolated sample, but based on a generally accepted property for the species, or anecdotal evidence). These evidence codes are from the Gene Ontology project [31]. If the evidence code is IDA, then the property was directly observed for a living isolate by one of the authors or an expert mentioned in the acknowledgements. 


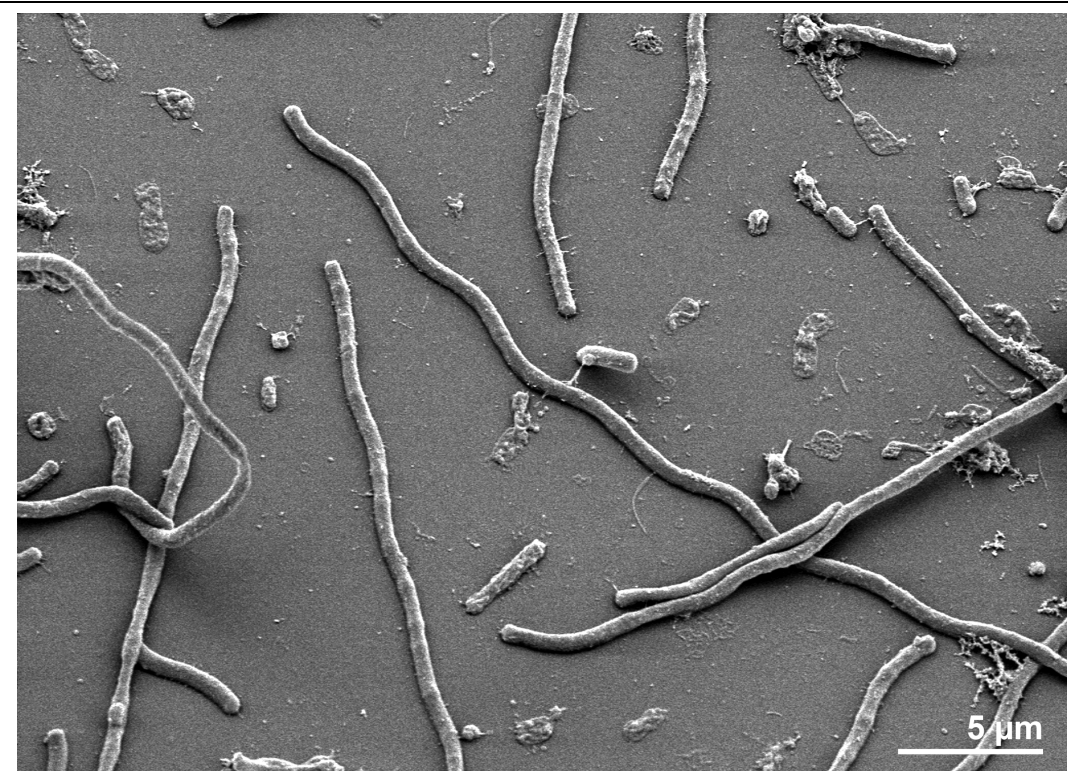

Figure 2. Scanning electron micrograph of $S$. grandis Sa g1

\section{Genome sequencing and annotation Genome project history}

This organism was selected for sequencing on the basis of its phylogenetic position [32], and is part of the Genomic Encyclopedia of Bacteria and Archaea project [33]. The genome project is deposited in the Genomes On Line Database [17] and the complete genome sequence is deposited in GenBank. Sequencing, finishing and annotation were performed by the DOE Joint Genome Institute (JGI). A summary of the project information is shown in Table 2.

\section{Growth conditions and DNA isolation}

S. grandis strain Sa g1, DSM 2844, was grown in DSMZ medium 172 (Cytophaga (marine) medium) [34] at $28^{\circ} \mathrm{C}$. DNA was isolated from $0.5-1 \mathrm{~g}$ of cell paste using Jetflex Genomic DNA Purification kit (GENOMED 600100) following the standard protocol as recommended by the manufacturer without modification. DNA will be available on request through the DNA Bank Network [46].

\section{Genome sequencing and assembly}

The genome was sequenced using a combination of Illumina and 454 sequencing platforms. All general aspects of library construction and sequencing can be found at the JGI website [35]. Pyrosequencing reads were assembled using the Newbler assembler (Roche). The initial Newbler assembly consisting of 551 contigs in six scaffolds was converted into a phrap [36] assembly by making fake reads from the consensus, to collect the read pairs in the 454 paired end library. Illumina GAii sequencing data $(3,575.7$ $\mathrm{Mb}$ ) was assembled with Velvet [37] and the consensus sequences were shredded into $1.5 \mathrm{~kb}$ overlapped fake reads and assembled together with the 454 data. The 454 draft assembly was based on $72.8 \mathrm{Mb}$ of 454 paired end data. Newbler parameters

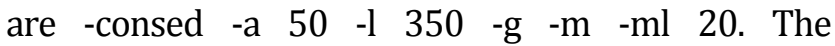
Phred/Phrap/Consed software package [36] was used for sequence assembly and quality assessment in the subsequent finishing process. After the shotgun stage, reads were assembled with parallel phrap (High Performance Software, LLC). Possible misassemblies were corrected with gapResolution [35], Dupfinisher [38], or sequencing cloned bridging PCR fragments with subcloning. Gaps between contigs were closed by editing in Consed, by PCR and by Bubble PCR primer walks (J.-F. Chang, unpublished). A total of 45 additional reactions were necessary to close gaps and to raise the quality of the final contigs. Illumina reads were also used to correct potential base errors and increase consensus quality using a software Polisher developed at JGI [39]. The final assembly consists of 84 contigs in 5 scaffolds. Together, the combination of the Illumina and 454 sequencing platforms provided $777.1 \times$ coverage of the genome. The final assembly contained 235,183 pyrosequence and 45,502,670 Illumina reads. 
Table 2. Genome sequencing project information

\begin{tabular}{lll}
\hline MIGS ID & Property & Term \\
\hline MIGS-31 & Finishing quality & Improved-High-Quality Draft \\
MIGS-28 & Libraries used & Two genomic libraries: one 454 PE library (9 kb insert size), one \\
MIGS-29 & Sequencing platforms & Illumina library \\
MIGS-31.2 & Sequencing coverage & $768.5 \times$ Illumina; 8.6 $\times$ pyrosequence \\
MIGS-30 & Assemblers & Newbler version 2.3, Velvet version 1.0.13, phrap version 1.080812 \\
MIGS-32 & Gene calling method & Prodigal 1.4, GenePRIM \\
& INSDC ID & requested February 9, 2012 \\
& GenBank Date of Release & Pending \\
& GOLD ID & Gi03955 \\
& NCBI project ID & 61003 \\
& Database: IMG-GEBA & 2509276035 \\
MIGS-13 & Source material identifier & DSM 2844 \\
& Project relevance & Tree of Life, GEBA \\
\hline
\end{tabular}

\section{Genome annotation}

Genes were identified using Prodigal [40] as part of the Oak Ridge National Laboratory genome annotation pipeline, followed by a round of manual curation using the JGI GenePRIMP pipeline [41]. The predicted CDSs were translated and used to search the National Center for Biotechnology Information (NCBI) non-redundant database, UniProt, TIGRFam, Pfam, PRIAM, KEGG, COG, and InterPro databases. Additional gene prediction analysis and functional annotation was performed within the Integrated Microbial Genomes - Expert Review (IMG-ER) platform [42].

\section{Genome properties}

The Improved-High-Quality draft assembly of the genome consists of 84 contigs in four scaffolds representing the chromosome $(4,422,561 \mathrm{bp}, 11,045$ bp, 2,786 bp and 2,223 bp length, respectively) and one 56,635 bp plasmid scaffold, with an overall $46.1 \% \mathrm{G}+\mathrm{C}$ content (Table 3 and Figure 3 ). Of the 3,598 genes predicted, 3,536 were protein-coding genes, and 62 RNAs; 70 pseudogenes were also identified. The majority of the protein-coding genes $(57.4 \%)$ were assigned a putative function while the remaining ones were annotated as hypothetical proteins. The distribution of genes into COGs functional categories is presented in Table 4.

Table 3. Genome Statistics

\begin{tabular}{lrr}
\hline Attribute & Value & \% of Total \\
\hline Genome size (bp) & $4,495,250$ & $100.00 \%$ \\
DNA coding region (bp) & $3,693,336$ & $82.16 \%$ \\
DNA G+C content (bp) & $2,067,067$ & $46.06 \%$ \\
Number of scaffolds & $5 *$ & \\
Extrachromosomal elements & 1 & \\
Total genes & 3,598 & $100.00 \%$ \\
RNA genes & 62 & $1.72 \%$ \\
rRNA operons & $3 * *$ & \\
tRNA genes & 48 & $1.33 \%$ \\
Protein-coding genes & 3,536 & $98.28 \%$ \\
Pseudo genes & 70 & $1.95 \%$ \\
Genes with function prediction (proteins) & 2,064 & $57.37 \%$ \\
Genes in paralog clusters & 1,575 & $43.77 \%$ \\
Genes assigned to COGs & 2,064 & $57.37 \%$ \\
Genes assigned Pfam domains & 2,072 & $57.59 \%$ \\
Genes with signal peptides & 1,109 & $30.82 \%$ \\
Genes with transmembrane helices & 687 & $19.09 \%$ \\
CRISPR repeats & 5 & \\
\hline
\end{tabular}

* four scaffolds for the chromosome and one for a plasmid

** only two rRNA operons appear to be complete; the third copy appears to be split into two incomplete fractions due to unresolved assembly problems. 

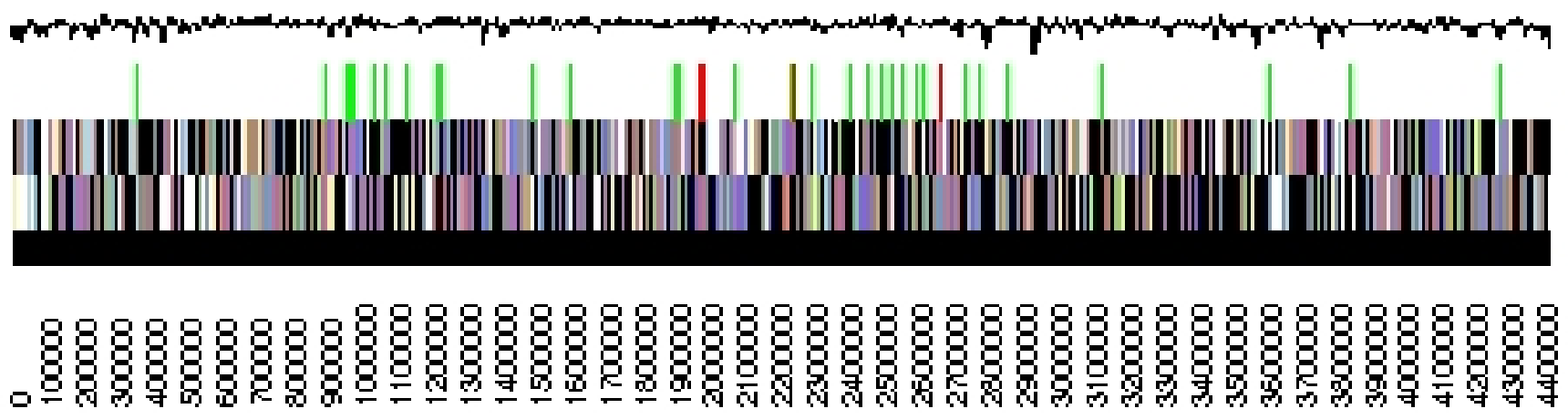

Figure 3. Graphical map of the largest scaffold, SapgrDRAFT_Contig123.4, which represents $>99.6 \%$ of the chromosome. The smaller contigs of the chromosome and the plasmid are not shown, but accessible through the img/er pages on the JGI web pages [35,42]. From bottom to top: Genes on forward strand (colored by COG categories), Genes on reverse strand (colored by COG categories), RNA genes (tRNAs green, rRNAs red, other RNAs black), GC content, GC skew.

Table 4. Number of genes associated with the general COG functional categories

\begin{tabular}{crrl}
\hline Code & value & \%age & Description \\
\hline J & 152 & 6.8 & Translation, ribosomal structure and biogenesis \\
A & 0 & 0.0 & RNA processing and modification \\
K & 118 & 5.3 & Transcription \\
L & 167 & 7.5 & Replication, recombination and repair \\
B & 1 & 0.0 & Chromatin structure and dynamics \\
D & 34 & 1.5 & Cell cycle control, cell division, chromosome partitioning \\
Y & 0 & 0.0 & Nuclear structure \\
V & 53 & 2.4 & Defense mechanisms \\
T & 98 & 4.4 & Signal transduction mechanisms \\
M & 217 & 9.7 & Cell wall/membrane biogenesis \\
N & 25 & 1.1 & Cell motility \\
Z & 1 & 0.0 & Cytoskeleton \\
W & 0 & 0.0 & Extracellular structures \\
U & 55 & 2.5 & Intracellular trafficking and secretion, and vesicular transport \\
O & 121 & 5.4 & Posttranslational modification, protein turnover, chaperones \\
C & 108 & 4.9 & Energy production and conversion \\
G & 51 & 2.3 & Carbohydrate transport and metabolism \\
E & 125 & 5.6 & Amino acid transport and metabolism \\
F & 57 & 2.6 & Nucleotide transport and metabolism \\
H & 101 & 4.5 & Coenzyme transport and metabolism \\
I & 88 & 4.0 & Lipid transport and metabolism \\
P & 91 & 4.1 & Inorganic ion transport and metabolism \\
Q & 35 & 1.6 & Secondary metabolites biosynthesis, transport and catabolism \\
R & 316 & 14.2 & General function prediction only \\
S & 215 & 9.7 & Function unknown \\
- & 1,534 & 42.6 & Not in COGs \\
\hline & & & \\
\hline
\end{tabular}




\section{Insights into the genome sequence}

\section{Comparison with the genome sequence of}

\section{S. grandis strain Lewin}

The two complete copies of the 16S rRNA gene in the Sa g1 genome show $99.5 \%$ sequence identity with those of strain Lewin [6], but only $98.0 \%$ sequence identity with the respective sequence from the yet to be genome-sequenced type strain Lewin $\mathrm{WH}^{\mathrm{T}}$ (ATCC 23119, M58795) [43]; this discrepancy is due to the huge number of ambiguous base calls in M58795, and is relativized by $99.4 \%$ identical bases within the HSPs. Given the different habitats of the two sequenced strains it is interesting to compare some basic genome features and their membership to the same species.

The second largest scaffold in the ImprovedHigh-Quality draft assembly of the Sa g1 genome (SapgrDRAFT_Contig162.5) has a size of 56,635 $\mathrm{bp}$, which is comparable to the size of plasmid SGRA01 in $S$. grandis strain Lewin, 54,948 bp (CP002832) [6]. A BLAST search against the NCBI $\mathrm{nr}$ database revealed a full length colinearity with about $94 \%$ sequence identity between SapgrDRAFT_Contig162.5 and the plasmid of $S$. grandis strain Lewin. Further comparison of the two sequences with the GGDC-Genome-toGenome Distance Calculator [44,45] revealed distances of only 0.0704 (formula 1 ) to 0.1342 (formula 3), corresponding to 82.7 to $88.3 \% \mathrm{DDH}$ values. SapgrDRAFT_3602 encodes a protein involved in initiation of plasmid replication, RepB, while the largest fraction of (13) genes encoded on this scaffold belong to COG function category 'nucleotide transport and metabolism' (similar to SGRA01); therefore suggesting that SapgrDRAFT_Contig162.5 is a plasmid whose sequence was not circularized during the genome assembly.

The largest scaffold in the draft assembly of Sa g1 (SapgrDRAFT_Contig123.4) has a size of $4,422,561 \mathrm{bp}$, which is comparable to the size of the $S$. grandis strain Lewin chromosome, $4,345,237 \mathrm{bp}$. The overall genome statistics (see Table 3) of the two strains is similar in some features, such as $\mathrm{G}+\mathrm{C}$ content $(46.1 \%$ strain Sa g1 vs. $46.4 \%$ strain Lewin), total number of genes $(3,598 v s .4,311)$, genes with function predictions (2,064 vs. 2,173), three rRNA operons (both), but deviates more in others, such as genes in paralog clusters $(1,575$ vs. 215), genes with signal peptides $(1,109$ vs. 589), and genes with transmembrane helices (687 vs. 778), which may reflect the differences in the gene calling and annotation process (strain Lewin is not yet featured in IMG [42]. As for the number of genes associated with the general COG functional categories (see Table 4) there are categories with very similar content, such as transcription (118 genes, both), translation (152 vs. 160), defense mechanisms (53vs. 52), cell motility (25 vs. 26), lipid transport (88 vs. 90) and cell wall/membrane biogenesis (217 vs. 206), while other categories deviate more significantly, such as replication (167 vs. 186), cell cycle control (34 vs. 20), intracellular trafficking (55 vs. 44), energy production and conversion (108 vs. 123), and secondary metabolites biosynthesis an catabolism (35 vs. 52), which again might be partially attributed to different procedures in the annotation processes.

The sequences of SapgrDRAFT_Contig123.4 and the chromosome of strain Lewin (CP002831), which represent roughly $99 \%$ of the respective genomes, were also compared with the GGDCGenome-to-Genome Distance Calculator $[44,45]$. The inferred distances from formulas 1 and 3 were 0.1139 and 0.1741 , respectively, corresponding to $83.1 \%$ and $77.9 \%$ DDH values, respectively, estimated via regression-based predictions. These values indicate that both strains belong to the same species, $S$. grandis.

The sequence of the three smaller scaffolds (SapgrDRAFT_Contig118.2 with 11,045 bp length, SapgrDRAFT_Contig106.1 with 2,786 bp and SapgrDRAFT_Contig119.3 with 2,223 bp) were compared against the NCBI nr database and revealed significant similarities only with the chromosome of strain Lewin. 


\section{Acknowledgements}

We would like to gratefully acknowledge the help of Maren Schröder (DSMZ) for growing $S$. grandis cultures. This work was performed under the auspices of the US Department of Energy Office of Science, Biological and Environmental Research Program, and by the University of California, Lawrence Berkeley National Laboratory under contract No. DE-AC02-05CH11231,

\section{References}

1. Dawyndt P, Vancanneyt M, De Meyer H, Swings J. Knowledge accumulation and resolution of data inconsistencies during the integration of microbial information sources. IEEE Trans Knowl Data Eng 2005; 17:1111-1126. http://dx.doi.org/10.1109/TKDE.2005.131

2. Garrity G. NamesforLife. BrowserTool takes expertise out of the database and puts it right in the browser. Microbiol Today 2010; 37:9.

3. Skerman VBD, McGowan V, Sneath PHA, eds. Approved Lists of Bacterial Names. Int J Syst Bacteriol 1980; 30:225-420. http://dx.doi.org/10.1099/00207713-30-1-225

4. Reichenbach H. Saprospira grandis (Leucotrichales) - Wachstum und Bewegung. Film E2424 des Instituts Wiss Film, Göttingen, Publ Wiss Film, Sekt Biol 1980; 13:26/E2424.

5. Euzéby JP. List of Beacterial Names with Stranding in Nomenclature: a folder on the internet. Int J Syst Bacteriol 1997; 47:590-592. PubMed http://dx.doi.org/10.1099/00207713-47-2-590

6. Saw JHW, Yuryew A, Kanbe M, Hou S, Young AG, Aizawa SI, Alam M. Complete genome sequencing and analysis of Saprospira grandis str. Lewin, a predatory marine bacterium. Stand $\mathrm{Ge}$ nomic Sci 2012; 6:84-93.

7. Altschul SF, Gish W, Miller W, Myers EW, Lipman DJ. Basic local alignment search tool. J Mol Biol 1990; 215:403-410. PubMed

8. Korf I, Yandell M, Bedell J. BLAST, O'Reilly, Sebastopol, 2003.

9. DeSantis TZ, Hugenholtz $\mathrm{P}$, Larsen N, Rojas $\mathrm{M}$, Brodie EL, Keller K, Huber T, Dalevi D, Hu P, Andersen GL. Greengenes, a chimera-checked $16 \mathrm{~S}$ rRNA gene database and workbench compatible with ARB. Appl Environ Microbiol 2006; 72:5069-5072. PubMed http://dx.doi.org/10.1128/AEM.03006-05
Lawrence Livermore National Laboratory under Contract No. DE-AC52-07NA27344, and Los Alamos National Laboratory under contract No. DE-AC0206NA25396, UT-Battelle and Oak Ridge National Laboratory under contract DE-AC05-000R22725, as well as German Research Foundation (DFG) INST 599/1-2.

10. Porter MF. An algorithm for suffix stripping. Program: electronic library and information systems 1980; 14:130-137.

11. Lee C, Grasso C, Sharlow MF. Multiple sequence alignment using partial order graphs. Bioinformatics 2002; 18:452-464. PubMed

http://dx.doi.org/10.1093/bioinformatics/18.3.452

12. Castresana J. Selection of conserved blocks from multiple alignments for their use in phylogenetic analysis. Mol Biol Evol 2000; 17:540-552. PubMed

http://dx.doi.org/10.1093/oxfordjournals.molbev.a $\underline{026334}$

13. Stamatakis $\mathrm{A}$, Hoover $\mathrm{P}$, Rougemont J. A rapid bootstrap algorithm for the RAxML web servers. Syst Biol 2008; 57:758-771. PubMed http://dx.doi.org/10.1080/10635150802429642

14. Hess PN, De Moraes Russo CA. An empirical test of the midpoint rooting method. Biol J Linn SoC Lond 2007; 92:669-674.

http://dx.doi.org/10.1111/j.1095$\underline{\text { 8312.2007.00864.x }}$

15. Pattengale ND, Alipour M, Bininda-Emonds ORP, Moret BME, Stamatakis A. How many bootstrap replicates are necessary? Lect Notes Comput Sci 2009; 5541:184-200. http://dx.doi.org/10.1007/978-3-642-02008-7_13

16. Swofford DL. PAUP*: Phylogenetic Analysis Using Parsimony (*and Other Methods), Version 4.0 b10. Sinauer Associates, Sunderland, 2002.

17. Pagani I, Liolios K, Jansson J, Chen IM, Smirnova T, Nosrat B, Markowitz VM, Kyrpides NC. The Genomes OnLine Database (GOLD) v.4: status of genomic and metagenomic projects and their associated metadata. Nucleic Acids Res 2012; 40:D571-D579. PubMed http://dx.doi.org/10.1093/nar/gkr1100

18. Daligault H, Lapidus A, Zyetun A, Nolan M, Lucas S, Glavina Del Rio T, Tice H, Cheng JF, Tapia $\mathrm{R}$, Han C, et al. Complete genome sequence of Haliscomenobacter hydrossis type strain $\left(\mathrm{O}^{\mathrm{T}}\right)$. 
Stand Genomic Sci 2011; 4:352-360. PubMed

http://dx.doi.org/10.4056/sigs.1964579

19. Field D, Garrity G, Gray T, Morrison N, Selengut J, Sterk P, Tatusova T, Thomson N, Allen MJ, Angiuoli SV, et al. The minimum information about a genome sequence (MIGS) specification. Nat Biotechnol 2008; 26:541-547. PubMed http://dx.doi.org/10.1038/nbt1360

20. Woese CR, Kandler O, Wheelis ML. Towards a natural system of organisms. Proposal for the domains Archaea and Bacteria. Proc Natl Acad Sci USA 1990; 87:4576-4579. PubMed http://dx.doi.org/10.1073/pnas.87.12.4576

21. Validation List No. 143. Int I Syst Evol Microbiol 2012; 62:1-4.

http://dx.doi.org/10.1099/ijs.0.039487-0

22. Krieg NR, Ludwig W, Euzéby J, Whitman WB. Phylum XIV. Bacteroidetes phyl. nov. In: Krieg NR, Staley JT, Brown DR, Hedlund BP, Paster BJ, Ward NL, Ludwig W, Whitman WB (eds), Bergey's Manual of Systematic Bacteriology, Second Edition, Volume 4, Springer, New York, 2011, p. 25.

23. Kämpfer P. Class III. Sphingobacteriia class. nov. In: Krieg NR, Staley JT, Brown DR, Hedlund BP, Paster BJ, Ward NL, Ludwig W, Whitman WB (eds), Bergey's Manual of Systematic Bacteriology, Second Edition, Volume 4, Springer, New York, 2011, p. 330.

24. Kämpfer P. Order I. Sphingobacteriales ord. nov. In: Krieg NR, Staley JT, Brown DR, Hedlund BP, Paster BJ, Ward NL, Ludwig W, Whitman WB (eds), Bergey's Manual of Systematic Bacteriology, Second Edition, Volume 4, Springer, New York, 2011.

25. Krieg NR, Staley JT, Brown DR, Hedlund BP, Paster BJ, Ward NL, Ludwig W, Whitman WB. FamiIy III. Saprospiraceae fam. nov. In: Krieg NR, Staley JT, Brown DR, Hedlund BP, Paster BJ, Ward NL, Ludwig W, Whitman WB (eds), Bergey's Manual of Systematic Bacteriology, Second Edition, Volume 4, Springer, New York, 2010, p. 358.

26. Gross J. Über freilebende Spironemaceen. Mitteilungen aus der Zoologischen Station zu Neapel 1911; 20:188-203.

27. Lewin RA, Leadbetter ER. Genus V. Saprospira Gross 1911, 190; Lewin 1962, 560 emend. mut. char. In: Buchanan RE, Gibbons NE (eds), Bergey's Manual of Determinative Bacteriology, Eighth Edition, The Williams and Wilkins Co., Baltimore, 1974, p. 109-111.
28. Lewin RA. Growth and nutrition of Saprospira grandis Gross (Flexibacterales). Can J Microbiol 1972; 18:361-365. PubMed http://dx.doi.org/10.1139/m72-055

29. Reichenbach $\mathrm{H}$. The Genus Saprospira. In: Dworkin M, Falkow, S, Rosenberg, E, Schleifer, $\mathrm{KH}$, Stackebrandt, E (eds). The Prokaryotes. 3rd ed. Volume 3. New York, NY: Springer; 2006. p 591-601.

30. BAuA. 2010, Classification of Bacteria and Archaea in risk groups. http://www.baua.de TRBA 466, p. 198.

31. Ashburner M, Ball CA, Blake JA, Botstein D, Butler H, Cherry JM, Davis AP, Dolinski K, Dwight SS, Eppig JT, et al. Gene ontology: tool for the unification of biology. The Gene Ontology Consortium. Nat Genet 2000; 25:25-29. PubMed http://dx.doi.org/10.1038/75556

32. Klenk HP, Göker M. En route to a genome-based classification of Archaea and Bacteria? Syst Appl Microbiol 2010; 33:175-182. PubMed http://dx.doi.org/10.1016/j.syapm.2010.03.003

33. Wu D, Hugenholtz P, Mavromatis K, Pukall R, Dalin E, Ivanova NN, Kunin V, Goodwin L, Wu $M$, Tindall BJ, et al. A phylogeny-driven Genomic Encyclopaedia of Bacteria and Archaea. Nature 2009; 462:1056-1060. PubMed http://dx.doi.org/10.1038/nature08656

34. List of growth media used at DSMZ: http://www.dsmz.de/catalogues/cataloguemicroorganisms/culture-technology/list-of-mediafor-microorganisms.html.

35. The DOE Joint Genome Institute. www.jgi.doe.gov

36. Phrap and Phred for Windows. MacOS, Linux, and Unix. www.phrap.com

37. Zerbino DR, Birney E. Velvet: algorithms for de novo short read assembly using de Bruijn graphs. Genome Res 2008; 18:821-829. PubMed http://dx.doi.org/10.1101/gr.074492.107

38. Han C, Chain P. Finishing repeat regions automatically with Dupfinisher. In: Proceeding of the 2006 international conference on bioinformatics \& computational biology. Arabnia HR, Valafar $\mathrm{H}$ (eds), CSREA Press. June 26-29, 2006: 141-146.

39. Lapidus A, LaButti K, Foster B, Lowry S, Trong S, Goltsman E. POLISHER: An effective tool for using ultra short reads in microbial genome assembly and finishing. AGBT, Marco Island, FL, 2008. 
Mavromatis et al.

40. Hyatt D, Chen GL, Locascio PF, Land ML, Larimer FW, Hauser LJ. Prodigal Prokaryotic Dynamic Programming Genefinding Algorithm. BMC Bioinformatics 2010; 11:119. PubMed http://dx.doi.org/10.1186/1471-2105-11-119

41. Pati A, Ivanova N, Mikhailova N, Ovchinikova G, Hooper SD, Lykidis A, Kyrpides NC. GenePRIMP: A Gene Prediction Improvement Pipeline for microbial genomes. Nat Methods 2010; 7:455-457. PubMed http://dx.doi.org/10.1038/nmeth.1457

42. Markowitz VM, Ivanova NN, Chen IMA, Chu K, Kyrpides NC. IMG ER: a system for microbial genome annotation expert review and curation. Bioinformatics 2009; 25:2271-2278. PubMed http://dx.doi.org/10.1093/bioinformatics/btp393

43. Gherna R, Woese CR. A partial phylogenetic analysis of the 'flavobacter-bacteroides' phylum: basis for taxonomic restructuring. Syst Appl Microbiol 1992; 15:513-521. PubMed
http://dx.doi.org/10.1016/S0723-2020(11)80110$\underline{4}$

44. Auch AF, von Jan M, Klenk HP, Göker M. Digital DNA-DNA hybridization for microbial species delineation by means of genome-to-genome sequence comparison. Stand Genomic Sci 2010; 2:117-134. PubMed http://dx.doi.org/10.4056/sigs.531120

45. Auch AF, Klenk HP, Göker M. Standard operating procedure for calculating genome-to-genome distances based on high-scoring segment pairs. Stand Genomic Sci 2010; 2:142-148. PubMed http://dx.doi.org/10.4056/sigs.541628

46. Gemeinholzer B, Dröge G, Zetzsche H, Haszprunar G, Klenk HP, Güntsch A, Berendsohn WG, Wägele JW. The DNA Bank Network: the start from a German initiative. Biopreserv Biobank 2011; 9:51-55.

http://dx.doi.org/10.1089/bio.2010.0029 